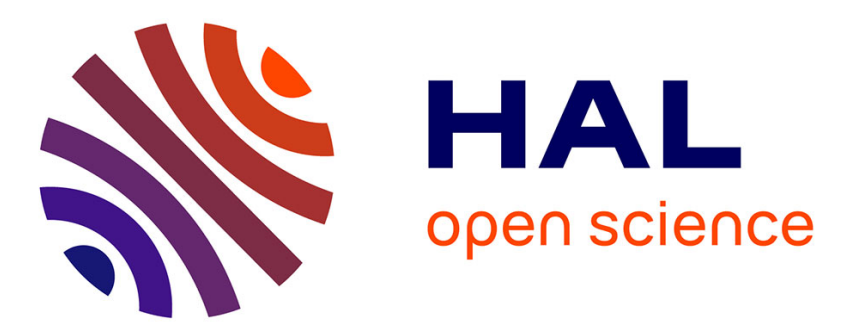

\title{
Multi-modular Algorithm for Computing the Splitting Field of a Polynomial
}

\author{
Guénaël Renault, Kazuhiro Yokoyama
}

\section{To cite this version:}

Guénaël Renault, Kazuhiro Yokoyama. Multi-modular Algorithm for Computing the Splitting Field of a Polynomial. ISSAC 2008 - 21st International Symposium on Symbolic and Algebraic Computation, Jul 2008, Linz/Hagenberg, Austria. pp.247-254, 10.1145/1390768.1390803 . hal-01305625

\section{HAL Id: hal-01305625 \\ https://hal.science/hal-01305625}

Submitted on 23 Nov 2016

HAL is a multi-disciplinary open access archive for the deposit and dissemination of scientific research documents, whether they are published or not. The documents may come from teaching and research institutions in France or abroad, or from public or private research centers.
L'archive ouverte pluridisciplinaire HAL, est destinée au dépôt et à la diffusion de documents scientifiques de niveau recherche, publiés ou non, émanant des établissements d'enseignement et de recherche français ou étrangers, des laboratoires publics ou privés. 


\section{Multi-modular Algorithm for Computing the Splitting Field of a Polynomial}

\author{
Guénaël Renault \\ INRIA, Paris-Rocquencourt, SALSA Project \\ UPMC, Univ. Paris 06, LIP6 \\ CNRS, UMR 7606, LIP6 \\ UFR Ingéniérie 919, LIP6 Passy-Kennedy, \\ Case 169, 4, Place Jussieu, F-75252 Paris \\ guenael.renault@lip6.fr
}

\author{
Kazuhiro Yokoyama \\ Rikkyo University \\ 3-34-1 Nishi lkebukuro, Toshima-ku \\ Tokyo 171-8501, Japan \\ yokoyama@rkmath.rikkyo.ac.jp
}

\begin{abstract}
Let $f$ be a univariate monic integral polynomial of degree $n$ and let $\left(\alpha_{1}, \ldots, \alpha_{n}\right)$ be an $n$-tuple of its roots in an algebraic closure $\overline{\mathbb{Q}}$ of $\mathbb{Q}$. Obtaining an algebraic representation of the splitting field $\mathbb{Q}\left(\alpha_{1}, \ldots, \alpha_{n}\right)$ of $f$ is a question of first importance in effective Galois theory. For instance, it allows us to manipulate symbolically the roots of $f$. In this paper, we propose a new method based on multi-modular strategy. Actually, we provide algorithms for this task which return a triangular set encoding the splitting ideal of $f$. We examine the ability/practicality of the method by experiments on a real computer and study its complexity.
\end{abstract}

\section{Categories and Subject Descriptors}

I.1 [Computing Methodologies]: Symbolic and algebraic manipulations

\section{General Terms}

Algorithms, Theory

\section{Keywords}

Galois theory, splitting field

\section{INTRODUCTION}

In [18] the authors proposed an approach for computing the splitting field of a monic integral polynomial $f$. This approach is based on indeterminate coefficients strategy and Hensel lifting. It takes as input the action of the Galois group of $f$ over approximation of roots of $f$ in a $p$-adic number field $\mathbb{Q}_{p}$ (or one of its extensions). To compute the Galois group $G_{f}$ of $f$ over $\mathbb{Q}$, the approach of $p$-adic approximation is very practical and efficient (see $[21,10,9]$ ).

In the approach of [18], the authors did not use all the data obtained during Galois group computation. Also, on some particular examples, the method was not so efficient. By experimentations, we discovered that using these data is a very low time consuming. Thus, in order to use a large part of these data and obtain a compromise for this approach we propose here a multi-modular approach for computing the splitting field of $f$. Moreover, the recent version 2.13 of the computer algebra system MAGMA [5] provides a new implementation (by Fieker and Klüners) of the Galois group computation based on $p$-adic approximations where it becomes easy to access the data computed during this procedure. Also, since the new computer architectures are now based on multi-core processors it is important to study new algorithms which can benefit from these new features. For all these reasons, a multi-modular strategy has to be studied.

The key of the multi-modular strategy proposed here comes from the following assertion: From data obtained during the computation of the permutation action of $G_{f}$ over approximate roots of $f$ modulo a prime $p_{1}$, we can easily obtain the action of the same permutation representation over approximate roots of $f$ modulo another prime $p_{2}$ (see Section $3)$.

From this action over approximate roots of $f$ modulo different primes we compute approximations, modulo the same primes, of the Gröbner basis $\mathcal{G}$ of the splitting ideal $\mathcal{M}$, that is the ideal of all the algebraic relations of the roots of $f$. Then we reconstruct it by Chinese Remainder Theorem (See Section 4). Thus, the splitting field of $f$ is given by $\mathbb{Q}\left[x_{1}, \ldots, x_{n}\right] / \mathcal{M}$; let us remark that it is easy to perform arithmetic operations in this algebra. Moreover, in general, expressions by primitive elements tend to suffer "expression swell", that is, huge coefficients appear and those harm the efficiency. So, for our purpose, simple extension does not seem suited.

In order to compute the approximate projections of $\mathcal{M}$ in different $p$-adic fields, we use the knowledge of certain algebraic structures, the action of $G_{f}$ over the $p$-adic approximation of roots of $f$ and a theoretical form of $\mathcal{G}$ given by the corresponding computation scheme, a very useful object introduced in [18] (see also [17]). The computation scheme gives sparse forms with indeterminate coefficients for the polynomials in $\mathcal{G}$ and techniques to avoid some computations (see Section 3.3). In Section 3 we show how to interpolate these sparse form by adaptation of the formulae given in [6] and modular computations. From these theoretical forms we deduce, in the same section, the best bounds 
in our knowledge for the coefficients of a basis of $\mathcal{M}$.

In Section 4 we give basic discussion on efficiency of multimodular strategy in a general form, and present concrete algorithms for our subject corresponding to this multi-modular strategy with effective tests for correctness. Then, based on the algorithms, we give certain results about the multimodular strategy's theoretical efficiency. Section 5 is devoted to the experiments, by which the practicality of the multi-modular strategy is examined.

\section{PRELIMINARIES}

We provide necessary notions and summarize some results of [21] and [18].

\subsection{Splitting Field and Galois Group over $\mathbb{Q}$}

Let $f(x)$ be a monic square-free integral polynomial of degree $n$ and $\alpha$ the set of all its roots in an algebraic closure $\overline{\mathbb{Q}}$ of $\mathbb{Q}$. The splitting field $K_{f}$ of $f$ is the extension field $\mathbb{Q}(\underline{\alpha})$ obtained by adjoining $\underline{\alpha}$ to $\mathbb{Q}$. The group $G_{f}$ of $\mathbb{Q}$-automorphisms of $K_{f}$ acts faithfully on $\underline{\alpha}$, thus one can consider the permutation representation $G_{f}$ of this group. Fixing a numbering of the roots $\underline{\alpha}=\left\{\alpha_{1}, \ldots, \alpha_{n}\right\}$ of $f, G_{f}$ is viewed as a subgroup of $S_{n}$. The group $G_{f}$ is called the Galois group of $f$.

To express $K_{f}$ symbolically, we consider the epimorphism $\phi: \mathbb{Q}\left[x_{1}, \ldots, x_{n}\right] \ni x_{i} \longmapsto \alpha_{i} \in K_{f}$ of $\mathbb{Q}$-algebras. For simplicity, we write $X=\left\{x_{1}, \ldots, x_{n}\right\}$. Then $K_{f}$ is represented by the residue class $\operatorname{ring} \mathcal{A}$ of the polynomial ring $\mathbb{Q}[X]$ factored by the kernel $\mathcal{M}$ of $\phi$. We call $\mathcal{M}$ the splitting ideal of $f$ associated with the assignment of the roots $\alpha_{1}, \ldots, \alpha_{n}$. In this setting, computing $K_{f}$ means to compute a Gröbner basis $\mathcal{G}$ of $\mathcal{M}$ (see [4]). Now we fix the lexicographic order $\prec$ on terms with $x_{1} \prec \cdots \prec x_{n}$, then the reduced Gröbner basis of $\mathcal{M}$ coincides with the generating set $\left\{g_{1}, g_{2}, \ldots, g_{n}\right\}$ obtained by successive extensions, that is, for each $i$,

1. $g_{i}$ is a polynomial in $x_{1}, \ldots, x_{i}$ and monic with respect to $x_{i}$, and

2. $\mathbb{Q}\left(\alpha_{1}, \ldots, \alpha_{i}\right) \cong \mathbb{Q}\left[x_{1}, \ldots, x_{i}\right] /\left\langle g_{1}, \ldots, g_{i}\right\rangle$,

where $\langle F\rangle$ denotes the ideal generated by an element or a set $F$. This implies that $g_{i}$ is an irreducible factor of $f\left(x_{i}\right)$ over $\mathbb{Q}\left[x_{1}, \ldots, x_{i-1}\right] /\left\langle g_{1}, \ldots, g_{i-1}\right\rangle$ such that $g_{i}\left(\alpha_{1}, \ldots, \alpha_{i}\right)=0$. Thus this reduced Gröbner basis can be obtained by "algebraic factoring methods" (see [3]) and is said to be a triangular basis (see $[12,6]$ ). For a Gröbner basis $\mathcal{G} \subset \mathbb{Q}[X]$ and a polynomial $P$, let $\operatorname{NF}(P, \mathcal{G})$ denote the normal form of $P$ in $\mathbb{Q}[X]$ with respect to $\mathcal{G}$ (see $[4]$ ).

The group $S_{n}$ acts naturally on $\mathbb{Q}[X]$ with $x_{i}^{\sigma}=x_{i}$ for $1 \leqslant i \leqslant n$ and $\sigma \in S_{n}$. Thus $G_{f}$ is the $\mathbb{Q}$-automorphisms group of $\mathcal{A}$ denoted by $\operatorname{Aut}_{\mathbb{Q}}(\mathcal{A})$ (see $\left.[3,1]\right)$. We use the following notation for groups: For a group $G$ acting on a set $\mathcal{S}$, the stabilizer in $G$ of an element or a subset $A$ of $\mathcal{S}$ is denoted by $\operatorname{Stab}_{G}(A)$, i.e. $\operatorname{Stab}_{G}(A)=\left\{\sigma \in G: A^{\sigma}=\right.$ $A\}$. If $G$ is the full symmetric group on $\mathcal{S}$, we simply write $\operatorname{Stab}(A)$ for $\operatorname{Stab}_{G}(A)$. We denote by $\operatorname{Stab}_{G}\left(\left[a_{1}, \ldots, a_{k}\right]\right)$ the point-wise stabilizer of a subset $A=\left\{a_{1}, \ldots, a_{k}\right\}$ of $\mathcal{S}$, i.e. $\operatorname{Stab}_{G}\left(\left[a_{1}, \ldots, a_{k}\right]\right)=\left\{\sigma \in G \mid a_{i}^{\sigma}=a_{i}, \forall i \in\{1, \ldots, k\}\right\}$. The set of right cosets of $H$ in $G$ is denoted by $H \backslash G$ and the set of all representatives of $H \backslash G$ by $H \backslash \backslash G$.

Definition 1 . We call the ideal generated by the polynomials $t_{1}+a_{1}, \ldots, t_{n}+(-1)^{n-1} a_{n}$, where $t_{i}$ is the $i$-th elementary symmetric function on $X$ and $f(x)=x^{n}+a_{1} x^{n-1}+$ $\cdots+a_{n}$, the universal splitting ideal of $f$ and denote it by $\mathcal{M}_{0}$. We call the residue class ring $\mathbb{Q}[X] / \mathcal{M}_{0}$ the universal splitting ring of $f$ over $\mathbb{Q}$ and denote it by $\mathcal{A}_{0}$.

With respect to the fixed order $\prec$, the reduced Gröbner basis of $\mathcal{M}_{0}$ is composed of the $n$ Cauchy's modules of $f$ (see [19]) and it is called the standard generating set. Since $S_{n}$ stabilizes $\mathcal{M}_{0}, S_{n}$ also acts faithfully on $\mathcal{A}_{0}$, i.e. $S_{n} \subset$ $\operatorname{Aut}_{\mathbb{Q}}\left(\mathcal{A}_{0}\right)$. We have the following theorem (see $[16,2,21]$ for details and other references).

THEOREM 2.1. There is a one-to-one correspondence between the set of all primitive idempotents of $\mathcal{A}_{0}$ and the set of all prime divisors of $\mathcal{M}_{0}$. Let $m$ be the primitive idempotent corresponding to the fixed prime divisor $\mathcal{M}$. Then, $G_{f}=\operatorname{Stab}(\mathcal{M})=\operatorname{Stab}(m)$ and $\mathcal{M}^{\sigma}=\left\{g \in \mathbb{Q}[X] \mid g m^{\sigma}=\right.$ 0 in $\left.\mathcal{A}_{0}\right\}$. Moreover, we have $\mathcal{M}_{0}=\cap_{\sigma \in G_{f} \backslash \backslash S_{n}} \mathcal{M}^{\sigma}$ and $\mathcal{A}_{0}=\oplus_{\sigma \in G_{f} \backslash \backslash S_{n}} m^{\sigma} \mathcal{A}_{0}=\oplus_{\sigma \in G_{f} \backslash \backslash S_{n}} \mathbb{Q}[X] / \mathcal{M}^{\sigma}$.

\subsection{Splitting Field and Modular Computation}

Now we consider the relation between the splitting ring over $\mathbb{Q}$ and that over a $p$-adic field $\mathbb{Q}_{p}$. The $n$-tuple $\alpha=$ $\left\{\alpha_{1}, \ldots, \alpha_{n}\right\}$ and the splitting ideal $\mathcal{M}$ associated with the assignment $x_{i}$ to $\alpha_{i}$ are fixed. The primitive idempotent of $\mathcal{A}_{0}$ corresponding to $\mathcal{M}$ is denoted by $m$. For a prime integer $p$, we denote by $\mathbf{Z}_{p}^{0}$ (resp. $\mathbf{Z}_{p}$ ) the localization of $\mathbf{Z}$ at $p$ (resp. the completion of $\mathbf{Z}_{p}^{0}$ ). We denote by $\pi_{p}$ the projection from $\mathbf{Z}_{p}[X]$ to $\mathbb{F}_{p}[X]$ (the natural extension of the projection from $\mathbf{Z}$ to $\mathbb{F}_{p}$ ). From now on, we will consider prime numbers $p$ satisfying the following property:

$$
\mathbb{P}: \quad \pi_{p}(f) \text { is square-free. }
$$

Let $\overline{\mathcal{M}}_{0}^{p}$ denote the ideal $\pi_{p}\left(\mathcal{M}_{0} \cap \mathbf{Z}_{p}^{0}[X]\right)$ in $\mathbb{F}_{p}[X]$ and $\mathcal{G}_{0}$ denote the standard generating set of $\mathcal{M}_{0}$. By construction, the Cauchy's modules of $f$ are polynomials with integral coefficients and monic in their greatest variable. Thus, the set $\pi_{p}\left(\mathcal{G}_{0}\right)$ is a Gröbner basis of $\overline{\mathcal{M}}_{0}^{p}$. Moreover, $\mathcal{G}_{0}$ is a Gröbner basis of the universal splitting ideal $\mathbb{Q}_{p} \otimes_{\mathbb{Q}} \mathcal{M}_{0}$ of $f$ as a polynomial with coefficients in $\mathbb{Q}_{p}$ and that of $\mathbf{Z}_{p}[X] \otimes \mathbf{Z}_{p}^{0}\left(\mathcal{M}_{0} \cap \mathbf{Z}_{p}^{0}[X]\right)$ over $\mathbf{Z}_{p}$. The ideal $\mathbb{Q}_{p} \otimes_{\mathbb{Q}} \mathcal{M}_{0}$ is denoted by $\mathcal{M}_{0}^{(p, \infty)}$. We denote $\mathbb{F}_{p}[X] / \overline{\mathcal{M}}_{0}^{p}$ by $\overline{\mathcal{A}}_{0}^{p}$ and $\mathbb{Q}_{p}[X] / \mathcal{M}_{0}^{(p, \infty)}$ by $\mathcal{A}_{0}^{(p, \infty)}$. We have the following result (see $[21,18])$.

THEOREM 2.2. We have the following assertions:

1. The projection $\pi_{p}$ gives a one-to-one correspondence between the set of all primitive idempotents of $\mathcal{A}_{0}^{(p, \infty)}$ and that of $\overline{\mathcal{A}}_{0}^{p}$. Moreover, for each pair $\left(\bar{m}^{(p)}, m^{(p, \infty)}\right)$ of corresponding primitive idempotents, $\operatorname{Stab}\left(\bar{m}^{(p)}\right)=\operatorname{Stab}\left(m^{(p, \infty)}\right)$.

2. The idempotent $m$ of $\mathcal{A}_{0}$ is also an idempotent of $\mathcal{A}_{0}^{(p, \infty)}$. Let $\bar{m}^{(p)}$ be a component of $\pi_{p}(m)$ and $m^{(p, \infty)}$ the primitive idempotent of $\mathcal{A}_{0}^{(p, \infty)}$ corresponding to $\bar{m}^{(p)}$. Then $\operatorname{Stab}(m)$ contains $\operatorname{Stab}\left(\bar{m}^{(p)}\right)\left(=\operatorname{Stab}\left(m^{(p, \infty)}\right)\right)$ and $\operatorname{Stab}\left(\pi_{p}(m)\right)=$ $\operatorname{Stab}(m)$. Moreover, if we denote $\operatorname{Stab}\left(\bar{m}^{(p)}\right) \backslash \backslash \operatorname{Stab}(m)$ by $\mathcal{S}$ then $\pi_{p}(m)=\sum_{\sigma \in \mathcal{S}} \bar{m}^{\sigma}$ and $m=\sum_{\sigma \in \mathcal{S}} m^{(p, \infty)^{\sigma}}$.

Now we fix a component $\bar{m}^{(p)}$ of $\pi_{p}(m)$ and its corresponding idempotent $m^{(p, \infty)}$ of $\mathcal{A}_{0}^{(p, \infty)}$. Let $\overline{\mathcal{M}}^{p}$ be the maximal ideal of $\mathbb{F}_{p}[X]$ corresponding to $\bar{m}^{p}$ and $\mathcal{M}^{(p, \infty)}$ the maximal ideal of $\mathbb{Q}_{p}[X]$ corresponding to $m^{(p, \infty)}$. Moreover, let $\mathcal{G}^{(p, \infty)}$ and $\overline{\mathcal{G}}^{(p)}$ be the reduced Gröbner basis of $\mathcal{M}^{(p, \infty)}$ and that of $\overline{\mathcal{M}}^{p}$ respectively. 
Definition 2. Let $\mathcal{G}^{(p, \infty)}=\left\{g_{1}^{(p, \infty)}, \ldots, g_{n}^{(p, \infty)}\right\}$. For a positive integer $k$, we call the polynomials set $\left\{g_{1}^{(p, \infty)} \bmod \right.$ $\left.p^{k+1}, \ldots, g_{n}^{(p, \infty)} \bmod p^{k+1}\right\}$ the $k$-th approximation to the basis $\mathcal{G}^{(p, \infty)}$ and denote it by $\mathcal{G}^{(p, k)}$. Note that $\mathcal{G}^{(p, 0)}=\overline{\mathcal{G}}^{(p)}$.

\section{Approximations of the roots of $f$}

The Gröbner basis $\overline{\mathcal{G}}^{(p)}$ can be lifted to $\mathcal{G}^{(p, \infty)}$ by Hensel construction based on quadratic iteration.

TheOREM 2.3. [21, 18] The reduced Gröbner basis $\mathcal{G}^{(p, \infty)}$ of the ideal $\mathcal{M}^{(p, \infty)}$ with respect to $\prec$ is contained in $\mathbf{Z}_{p}[X]$, and $\overline{\mathcal{G}}^{(p)}$ is lifted uniquely to $\mathcal{G}^{(p, \infty)}$ by Hensel construction.

REMARK 3. From $\overline{\mathcal{G}}^{(p)}$, we can construct the approximate Gröbner basis $\mathcal{G}^{(p, k)}$ for any integer $k$. As soon as we have $\mathcal{G}^{(p, k)}$, we can compute with the roots of $f$ in $\mathbb{Z} / p^{k+1} \mathbb{Z}$ by computing normal forms modulo this basis. Thus, in the sequel, the expression approximations of the roots of $f$ modulo $p^{p+1}$ will mean that we have such a Gröbner basis $\mathcal{G}^{(p, k)}$

REMARK 4. As to the Gröbner basis $\mathcal{G}$, the denominators of its elements are related to the discriminant $d(f)$ of $f$. (See Section 3.4.) For each prime $p$ satisfying the property $\mathbb{P}$, the square-freeness of $\pi_{p}(f)$ implies $\pi_{p}(d(f)) \neq 0$, and thus, it follows that $\pi_{p}(\mathcal{G})$ is well-defined, that is, $p$ does not divide any of the denominators.

Now we will study the construction of $\mathcal{G}$ by Chinese Remainder Theorem.

\section{MODULAR CONSTRUCTION OF $\mathcal{G}$}

In this section, we fix a splitting ideal $\mathcal{M}$ of $f$, the corresponding idempotent $m$ and its stabilizer, the Galois group $G_{f}$ represented as a sub-group of $S_{n}$. We denote by $Z_{\mathbb{K}}(I)$ the algebraic variety over a field $\mathbb{K}$ associated to an ideal $I$ in a polynomial ring. Let $E=\left\{e_{1}<\cdots<e_{s}\right\}$ be a subset of $\{1, \ldots, n\}$ and $\gamma=\left(\gamma_{1}, \ldots, \gamma_{n}\right)$ an element of $Z_{\overline{\mathbb{Q}}}(\mathcal{M})$. We denote by $\gamma(E)$ the projection of $\gamma$ on the indexes given by $E$ (i.e. $\left.\left(\gamma_{e_{1}}, \ldots, \gamma_{e_{s}}\right)\right)$ and $Z_{\overline{\mathbb{Q}}}(\mathcal{M})(E)=\{\underline{\gamma}(E): \underline{\gamma} \in$ $\left.Z_{\overline{\mathbb{Q}}}(\mathcal{M})\right\}$

\subsection{Approximation of $\mathcal{G}$}

Let $\mathcal{G}=\left\{g_{1}, \ldots, g_{n}\right\}$ be the Gröbner basis of $\mathcal{M}$, we will describe here how to compute the coefficients of polynomials $g_{i}$ 's by indeterminate coefficient strategy and multi-modular computation.

More precisely, by the knowledge of a special subset $E_{i}$ of $\{1, \ldots, n\}$, we can deduce an equation which defines the polynomial $g_{i}$ (see Section 3.3):

$$
g_{i}(\underline{\gamma})=0 \text { for every } \underline{\gamma} \in Z_{\overline{\mathbb{Q}}}(\mathcal{M})\left(E_{i}\right) .
$$

We can replace the variety $Z_{\overline{\mathbb{Q}}}(\mathcal{M})\left(E_{i}\right)$ by $Z_{\overline{\mathbb{Q}}_{p}}\left(\mathbb{Q}_{p} \otimes_{\mathbb{Q}} \mathcal{M}\right)\left(E_{i}\right)$ where $p$ is a prime satisfying $\mathbb{P}$. Then, by using approximations of roots, we obtain the same equation but for the approximation of $g_{i}$. To do that, we need to recall some results (see $[21]$ ).

Let $p$ be a prime integer satisfying $\mathbb{P}, \bar{m}^{(p)}$ a component of $\pi_{p}(m), \overline{\mathcal{M}}^{p}$ its corresponding maximal ideal of $\mathbb{F}_{p}[X]$ and $\mathcal{M}^{(p, \infty)}$ its corresponding maximal ideal of $\mathbb{Q}_{p}[X]$ which is a divisor of $\mathbb{Q}_{p}[X] \otimes_{\mathbb{Q}} \mathcal{M}$.

Proposition 3.1. Let $\mathcal{S}=\operatorname{Stab}\left(\bar{m}^{(p)}\right) \backslash \backslash \operatorname{Stab}(m)$. Then $\mathbb{Q}_{p} \otimes_{\mathbb{Q}} \mathcal{M}=\cap_{\sigma \in \mathcal{S}}\left(\mathcal{M}^{(p, \infty)}\right)^{\sigma}$, and $\pi_{p}\left(\mathcal{M} \cap \mathbf{Z}_{p}^{0}\right)=\cap_{\sigma \in \mathcal{S}}(\overline{\mathcal{M}})^{\sigma}$.
By Proposition 3.1, we can reduce the equation (3.1) to the following.

$$
\operatorname{NF}\left(g_{i},\left(\mathcal{G}^{(p, \infty)}\right)^{\sigma}\right)=0 \text { for every } \sigma \in G_{E_{i}} \backslash \backslash G_{f},
$$

where $G_{f}=\operatorname{Stab}(m)$ and $G_{E_{i}}$ denotes $\operatorname{Stab}\left(\left[\alpha_{e_{i, 1}}, \ldots, \alpha_{e_{i, t}}\right]\right)$ for $E_{i}=\left\{e_{i, 1}, \ldots, e_{i, t}\right\}$. Because $\left\{\left(\mathcal{G}^{(p, \infty)}\right)^{\sigma}: \sigma \in G_{E_{i}} \backslash \backslash G_{f}\right\}$ provides all elements of $Z_{\overline{\mathbb{Q}}_{p}}\left(\mathbb{Q}_{p} \otimes \mathbb{Q} \mathcal{M}\right)\left(E_{i}\right)$. Moreover, replacing $\mathcal{G}^{(p, \infty)}$ with $\mathcal{G}^{(p, k)}$ (see Remark 3), we have the following equation that approximation $g_{i} \bmod p^{k+1}$ must satisfy.

$$
\mathrm{NF}\left(g_{i},\left(\mathcal{G}^{(p, k)}\right)^{\sigma}\right) \equiv 0 \quad\left(\bmod p^{k+1}\right) \forall \sigma \in G_{E_{i}} \backslash \backslash G_{f} .
$$

REMARK 5. The components of $\pi_{p}(m)$ are conjugate to each other by the action of $G_{f}=\operatorname{Stab}(m)$. From this fact, it follows that any choice of $\bar{m}^{(p)}$ from the components of $\pi_{p}(m)$ still give the same Gröbner basis $\mathcal{G}=\left\{g_{1}, \ldots, g_{n}\right\}$.

In [18] the authors presented a linear system resolution to compute $g_{i} \bmod p^{k+1}$. Here, we will present in a further section how to do this by interpolation. Before that, we present how to compute an approximation of $g_{i}$ modulo an integer $M$ by Chinese Remainder Theorem.

\subsection{Chinese Remainder Construction}

Recall that $m$ is the idempotent of $\mathcal{A}_{0}$ corresponding to the fixed splitting ideal $\mathcal{M}$ corresponding to the specific roots ordering. We now consider primes $p_{1}, \ldots, p_{i}$ satisfying the property $\mathbb{P}$ and a component $\bar{m}^{\left(p_{i}\right)}$ of $\pi_{p_{i}}(m)$ for each $p_{i}$. Let $\mathcal{G}^{\left(p_{1}, k_{1}\right)}, \ldots, \mathcal{G}^{\left(p_{i}, k_{i}\right)}$ the approximate Gröbner bases corresponding to these components.

As seen in Section 3.1, we can approximate the polynomials $g_{i}$ modulo each $p_{i}^{k_{i}}$. Thus, by Chinese Remainder Theorem we lift them in the ring $\mathbb{Z} / M \mathbb{Z}$ where $M=\prod_{j=1}^{i} p_{j}^{k_{j}}$ From this computation we obtain the projection of $\mathcal{G} \bmod -$ ulo $M$, that is the set of polynomials $\left\{g_{1} \bmod M, \ldots, g_{n}\right.$ $\bmod M\}$. But, in all this computation we assume that the idempotent $m$ is fixed. In practice, we can not assume this hypothesis. Thus we need a general method to assure that each component $\bar{m}^{\left(p_{j}\right)}$ will correspond to the same idempotent $m$. (See Remark 5.)

To do that, we will use data produced during the computation of the Galois group $G_{f}$ done modulo $p_{1}^{k_{1}}$. Then we reorder the roots modulo primes $p_{2}, \ldots, p_{i}$ by following criteria obtained from the data. From the computation of $G_{f}$ $[21,10]$, we obtain a finite sequence $\left\{\left(I_{i}, A_{i}\right): i=1, \ldots, t\right\}$ of invariants and their integer evaluation modulo $p_{1}^{k_{1}}$ such that $N F\left(I_{i}-A_{i}, \mathcal{G}^{\left(p_{1}, k_{1}\right)}\right) \equiv 0\left(\bmod p_{1}^{k_{1}+1}\right)$ for every $i$. Since $p_{1}^{k_{1}}$ exceeds the computed theoretical bound so that the corresponding relative Lagrange resolvent has $A_{i}$ as its simple integral root. This implies $\left(I_{i}-A_{i}\right) m=0$ for every $i$ and $N F\left(g_{i}, \mathcal{G}^{\left(p_{1}, k_{1}\right)}\right) \equiv 0\left(\bmod p_{1}^{k_{1}+1}\right)$ for every $g_{i}$. Conversely, tracing the determination process of $G_{f}$ with different modulus $q^{k}$, if $q^{k}$ exceeds the bound, we have the same result. As $p_{1}^{k_{1}}$ already exceeds the bound, we have

THEOREM 3.2. Let $q$ be a prime satisfying the property $\mathbb{P}, \mathcal{G}^{(q, k)}$ be the $k$-th approximation of a Gröbner basis of a maximal divisor of $\mathbb{Q}_{q} \otimes_{\mathbb{Q}} \mathcal{M}_{0}$ and $\bar{m}^{(q)}$ its corresponding primitive idempotent of $\overline{\mathcal{M}}_{0}^{q}$. If $q^{k}>p_{1}^{k_{1}}$ and $N F\left(I_{i}-\right.$ $\left.A_{i}, \mathcal{G}^{(q, k)}\right) \equiv 0\left(\bmod q^{k+1}\right)$ for every $i$, then $N F\left(g_{i}, \mathcal{G}^{(q, k)}\right) \equiv$ $0\left(\bmod q^{k+1}\right)$ for every $g_{i}$, that is, $\bar{m}^{(q)}$ is a component of $\pi_{q}(m)$. 
This method can be seen as a modular Galois group computation guided by the knowledge of the exact branch taken during the descent from $S_{n}$ to $G_{f}$ in the permutations subgroups tree of degree $n$. Efficient implementations of Galois group computation use some techniques to cut this descending branch and allow to begin from a subgroup of $S_{n}$ (see [10]). To be more efficient, we plan to adapt our method with these techniques.

\subsection{Computation Scheme and $i$-relations}

In this section, we recall the definition and give some new results about computation scheme and $i$-relation (see [18]).

In $[18$, Section 3] the authors present a framework for the computation of the Gröbner basis $\mathcal{G}=\left\{g_{1}, \ldots, g_{n}\right\}$ with indeterminate coefficients strategy. In this framework, we attache to a particular permutation representation $G_{f}$ a set of good theoretical form for polynomials of $\mathcal{G}$ and techniques which allow us to avoid computations for some $g_{i}$. This is what we call computation scheme since this guides the algorithm for computing $\mathcal{G}$.

In particular, we associate to each polynomial $g_{i}$ an integers set $E_{i}=\left\{e_{1}<\cdots<e_{s}=i\right\}$ which describes a triangular set $T_{i}=\left\{g_{1}^{*}, \ldots, g_{s}^{*}=g_{i}\right\}$ where $g_{k}^{*} \in \mathbb{Q}\left[x_{e_{1}}, \ldots, x_{e_{k}}\right]$ and $g_{k}^{*}\left(x_{e_{k}}, \alpha_{e_{k-1}}, \ldots, \alpha_{e_{1}}\right)$ is a minimal polynomial of the $\mathbb{K}$-extension $\mathbb{K}\left(\alpha_{e_{k}}\right)$ where $\mathbb{K}=\mathbb{Q}\left(\alpha_{e_{k-1}}, \ldots, \alpha_{e_{1}}\right)$; we will denote by $d\left(E_{i}\right)_{k}$ the degree of this extension. In [18] the theoretical form $g_{s}^{*}$ is used to compute $g_{i}$ by indeterminate coefficients strategy. The number of coefficients to compute is deduced from $E_{i}$ (or equivalently by $T_{i}$ ) and is denoted by $d\left(E_{i}\right)$ :

$$
d\left(E_{i}\right):=\prod_{k=1}^{s} \operatorname{deg}_{x_{e_{k}}} g_{k}^{*},
$$

and this quantity is called the degree of $E_{i}$.

There may be a lot of different sets $E_{i}$ which all correspond to the polynomial $g_{i}$ but, the smaller $d\left(E_{i}\right)$ is, the more efficient our algorithm will be. For example, we can choose the trivial set $E_{i}=\{1,2, \ldots, i-1, i\}$ which has maximal degree but in almost all cases we can find a better set $E_{i}$ corresponding to $g_{i}$. This is why these sets $E_{i}$ are important in our algorithm and we call them $i$-relations.

The computation scheme introduced in [18] provides also some techniques to avoid the computation of some $g_{i}$ 's. Thus, to this framework we attach the set $\mathcal{I}$ of integers corresponding to the polynomials we have to compute. The total number of coefficients to compute in $\mathcal{G}$ is the sum of the degrees of $i$-relation with $i$ in $\mathcal{I}$ and we denote it $c\left(G_{f}\right)$. To compute the polynomials with index in $\mathcal{I}$ modulo a power of a prime, the strategy used was based on indeterminate coefficients followed by a linear algebra step. Here we want to replace the second step by an interpolation step. This is what we present in the next section.

\subsection{Lagrange Formulae and $i$-relations}

In [6], Lagrange formulae are presented for general triangular sets. These formulae can be used to compute the Gröbner basis $\mathcal{G}$, this is what is done in [13]. In this case, the total number of coefficients to compute will be of the order of the size of the Galois group $G_{f}$ which may be very large. Thus, to overcome this problem we introduce Lagrange formulae for $i$-relations in order to use the computation scheme.

Let $E_{i}=\left\{e_{1}<\cdots<e_{s}=i\right\}$ be an $i$-relation and $T_{i}$ its associated triangular basis as defined in Section 3.3. The affine variety $Z_{\overline{\mathbb{Q}}}\left(T_{i}\right)$ is equiprojectable, thus one can apply the Lagrange formulae given in [6] but, since here we are in a very special context, we will restate the construction by using the permutation representation of the given Galois group $G_{f}$.

Let $\sigma$ be a given permutation in $S_{n}$. (Here we write $\sigma(a)$ for $a^{\sigma}$ for simplifying formulas.) We denote by $\mathcal{O}(j, \sigma)$ the orbit of $\sigma\left(e_{j}\right)$ under the action of the point-wise stabilizer $\operatorname{Stab}_{G_{f}}\left[\sigma\left(e_{1}\right), \sigma\left(e_{2}\right), \ldots, \sigma\left(e_{j-1}\right)\right]$ defined by $\left\{\tau \in G_{f}\right.$ $\left.\tau\left(\sigma\left(e_{i}\right)\right)=\sigma\left(e_{i}\right), \forall i \in\{1, \ldots, j-1\}\right\}$. By using the map $i \mapsto \alpha_{i}$, the set $\mathcal{O}(j, \sigma)$ corresponds to the orbit of the element $\sigma\left(\alpha_{e_{j}}\right)$ over the field $\mathbb{Q}\left(\alpha_{\sigma\left(e_{1}\right)}, \alpha_{\sigma\left(e_{2}\right)}, \ldots, \alpha_{\sigma\left(e_{j-1}\right)}\right)$.

From this orbit we can interpret the formula given in [6] in our specific case.

THEOREM 3.3. Let $E_{i}=\left\{e_{1}<\cdots<e_{s}=i\right\}$ be an $i$-relation. The corresponding polynomial $g_{i}$ verify $g_{i}=\mathcal{L}_{i}$ with

$\mathcal{L}_{i}=\sum_{\sigma \in \operatorname{Trans}}\left(\left(\prod_{j=1}^{s-1} \prod_{\substack{c \in O(j, \sigma) \\ e \neq \sigma\left(e_{j}\right)}} \frac{x_{e_{j}}-\alpha_{e}}{\alpha_{\sigma\left(e_{j}\right)}-\alpha_{e}}\right) \prod_{e \in O(s, \sigma)} x_{i}-\alpha_{e}\right)$

where Trans is the transversal $\operatorname{Stab}_{G_{f}}\left(\left[e_{1}, \ldots, e_{s}\right]\right) \backslash \backslash G_{f}$

\subsection{Bound for the Coefficients of $g_{s}^{*}$}

In the formula $\mathcal{L}_{i}$ given in Theorem 3.3 the denominators can be canceled by multiplying with a sufficiently large power of $d(f)$ the discriminant of the polynomial $f$. The multiplication by $d(f)$ can cancel two denominators of the form $\prod_{\substack{e \in O(j, \sigma) \\ e \neq \sigma\left(e_{j}\right)}} \frac{1}{\alpha_{\sigma\left(e_{j}\right)}-\alpha_{e}}$. Thus, all the denominators can be canceled by multiplying $\mathcal{L}_{i}$ with $D_{i}=d(f)^{\left\lceil\frac{s}{2}\right\rceil}$.

The polynomial $D_{i} \mathcal{L}_{i}$ has integral coefficients, we will now investigate a bound over the coefficient $c$ corresponding to the multi-degree $\left(k_{1}, \ldots, k_{s}\right)$. We denote by $d_{j}$ the degree in $x_{j}$ of $\mathcal{L}_{i}$. We note $d\left(E_{i}\right)=\prod_{k=1}^{s} d_{k}=\mid G_{f}$ : $S t a b_{G_{f}}\left(\left[e_{1}, \ldots, e_{s}\right]\right) \mid$. Let $\delta$ be a bound over the differences of roots $\left|\alpha_{i}-\alpha_{j}\right|$ and $\nu$ a bound over the absolute values of the roots $\left|\alpha_{i}\right|$. Here we will modify the proof given in [13] to deal with the case of an $i$-relation:

1. After cancellation of the denominators by multiplying with $D_{i}$, it remains, in the numerator, a product of $n(n-$ 1) $\left\lceil\frac{s}{2}\right\rceil-d_{1}-\ldots-d_{s}+s$ elements of the form $\left(\alpha_{j}-\alpha_{i}\right)$. This product will be distributed on all the coefficients of $g_{i}$ and is bounded by $\mathbb{B}=\delta^{n(n-1)\left\lceil\frac{s}{2}\right\rceil-d_{1}-\ldots-d_{s}+s}$.

2. The indeterminate $x_{e_{i}}$ of degree $k_{i}$ in $\mathcal{L}_{i}$ comes from a product of $d_{i}-1$ elements of the form $\left(x_{e_{i}}-\alpha_{j}\right)$. Thus, its absolute value can be bounded by the well known binomial quantity $\left(\begin{array}{c}d_{i}-1 \\ k_{i}\end{array}\right) \nu^{d_{i}-1-k_{i}}$.

Hence, by summing all these products over the transversal (see Theorem 3.3), we obtain the following bound for the absolute value of $c$ :

$$
d\left(E_{i}\right)\left(\begin{array}{c}
d_{1}-1 \\
k_{1}
\end{array}\right) \nu^{d_{1}-1-k_{1}} \ldots\left(\begin{array}{l}
d_{s} \\
k_{s}
\end{array}\right) \nu^{d_{s}-k_{s}} \mathbb{B}
$$

\section{MULTI-MODULAR STRATEGY}

In order to attain efficient computation of splitting fields, we can make good use of more sophisticated modular computation technique, "multi-modular" one. Here we show details on our technique and its variants for improvements.

\subsection{Basic Discussion on Modular Techniques}


There are several strategies on applying modular techniques for splitting field computation. Among those, a multimodular strategy described below shall be effective and efficient under the following assumption which seems natural phenomena for our problem.

Assumption: The computed theoretical bound, say $B_{T}$, on the coefficients of the Gröbner basis $\mathcal{G}$ is much larger than the real bound $B_{R}$, that is, the maximal absolute value of numerators and denominators of coefficients of $\mathcal{G}$. Also $B_{T}$ is much larger than the bound $B_{G}$ used for the Galois group determination.

Under the assumption, it is quite natural to use some heuristic bound $B_{H}$ much smaller than $B_{T}$. Our computation can be one instance of the following model:

Computational Model with Modular Computation Here, the target which we want to compute is some mathematical object over the rational number field $\mathbb{Q}$.

\section{Step 1. Candidate Computation: Proc_CAND}

Step 1-1. Modular Image Computation We set the modulus $q$, and then compute the modular image of the target modulo $q$.

Step 1-2. Conversion

By rational reconstruction, we have a candidate of the target.

\section{Step 2. Correctness Check: Proc_CHECK}

We check whether a candidate is correct or not by some efficiently computable test. If the test is OK, we have the correct result.

In our case, Proc_CHECK can be executed by ideal inclusion test, which shall correspond to trial division for polynomial factorization, as pointed out in [18]. Also, we may use another modular technique for Proc_CHECK. Further discussion will be given later.

When we use $2 B_{T}^{2}$ for the modulus, the computed candidate is always correct and Proc_CHECK is not necessary. (We note that for rational reconstruction, the modulus should be twice of square of the bound. ) On the other hand, when we use some heuristic modulus $q$, we have to execute recursive computation to reach the correct answer. When Proc_CHECK fails, we can apply several strategies in Step 1:

S1: Replace a larger modulus $q^{\prime}$ and execute Step 1-1.

S2: Take another modulus $q^{\prime}$ prime to $q$, execute Step 1-1 with $q^{\prime}$ and combine the result with the old one by Chinese remainder theorem. Then the modulus in Proc_CHECK becomes $q \times q^{\prime}$.

S3: Lift up the candidate to a larger modulus $q^{\prime}$ by Hensel construction.

Here, we call the strategy S2 multi-modular strategy, and the strategy S3 p-adic strategy. Apparently, the strategy S1 is not efficient compared with other two.

To find the most practical one among strategies in the above, we examine those total times of computation. Let $T_{P}(q)$ be the time of Step 1-1 of Proc_CAND, where $q$ is the modulus used, $T_{R}(q)$ the time of Step 1-2 and $T_{C}$ the time of Proc_CHECK. Here, we dare to omit the effect of $c\left(G_{f}\right)$ on those in order to make our argument clear. In Section 4.3, we will give further discussion taking $c\left(G_{f}\right)$ into account.

If we use $2 B_{T}^{2}$ for the modulus, Proc_CHECK is not necessary, and thus the total time $\left.T_{0}=T_{P}\left(2 B_{T}^{2}\right)+T_{R}\left(2 B_{T}^{2}\right)\right)$. If we use some heuristic modulus $q$, then we should repeat the computation till the modulus exceeds $2 B_{R}^{2}$. Suppose that we reach to it by $s$ times recursion, where $q_{1}, \ldots, q_{s}$ are moduli used. Then we have the following total times:

S2: $T_{2}=\sum_{i=1}^{s}\left(T_{P}\left(q_{i}\right)+T_{C R T}\left(q_{1} \cdots q_{i-1}, q_{i}\right)+T_{R}\left(\prod_{j=1}^{i} q_{j}\right)+\right.$ $\left.T_{C}\right)$, where $q_{1} \cdots q_{s-1}<2 B_{R}^{2}<q_{1} \cdots q_{s}$ and $T_{C R T}\left(q, q^{\prime}\right)$ denotes the time for Chinese remainder theorem for two moduli $q$ to $q^{\prime}$.

S3: $T_{3}=T_{P}\left(q_{1}\right)+\sum_{i=2}^{s}\left(T_{H}\left(q_{i}, q_{i-1}\right)+T_{R}\left(q_{i}\right)+T_{C}\right)$, where $q_{s-1}<2 B_{R}^{2}<q_{s}$ and $T_{H}\left(q^{\prime}, q\right)$ denotes the time for Hensel lifting from the modulus $q$ to $q^{\prime}$.

Getting precise estimation of $T_{C}$ is very difficult, when we apply ideal inclusion test. Thus, we may also apply additional modular technique to have efficient realization of Proc_CHECK. The basic procedure is the following:

Modular Check: Proc_modCHECK

Once we have a candidate $\mathcal{C}$ constructed by using modulo $q$, we check if it is still valid modulo another $q^{\prime}$. If so, we can show that $\mathcal{C}$ is still a candidate modulo $q \times q^{\prime}$. Otherwise, we compute the modular image of a candidate modulo $q^{\prime}$ by Step 1-1 of Proc_CAND and apply Chinese remainder theorem to get the modular image of new candidate $\mathcal{C}^{\prime}$ modulo $q \times q^{\prime}$.

Now we denote by $T_{M C}(q)$ the time for modular check modulo $q$, not including any candidate construction. In our case, we suppose that $T_{M C}(q)$ is much smaller than $T_{P}(q)$ and $T_{H}\left(q^{\prime}, q^{\prime \prime}\right)$ with $q=q^{\prime} / q^{\prime \prime}$. (See Section 4.3 for details.) Then, we have two types of usage of Modular CHECK:

U1: We can reduce the number of Proc_CHECK by repeating Proc_MODCHECK until we have a stable result. Then, it is highly supposed that the computed candidate is correct. With this practical assumption, the size of the total modulus is supposed the same order as that of $2 B_{R}^{2}$, and so that of $B_{R}$. Then we have $T_{2}=O\left(\sum_{i=1}^{s}\left(T_{P}\left(q_{i}\right)+\right.\right.$ $\left.\left.T_{C R T}\left(q_{1} \cdots q_{i-1}, q_{i}\right)+T_{R}\left(\prod_{j=1}^{i} q_{j}\right)+T_{M C}\left(q_{i}\right)\right)+T_{C}\right)$, where $\prod_{i=1}^{s} q_{i}=O\left(B_{R}\right)$, and $T_{3}=O\left(T_{P}\left(q_{1}\right)+\sum_{i=2}^{s}\left(T_{H}\left(q_{i}, q_{i-1}\right)\right.\right.$ $\left.\left.+T_{R}\left(q_{i}\right)+T_{M C}\left(q_{i}\right)\right)+T_{C}\right)$, where $q_{s}=O\left(B_{R}\right)$.

U2: We repeat Proc_MODCHECK till the total modulus reaches the theoretical bound. (So, the p-adic strategy is not suited for this approach.) This may sound somehow contradictory to our strategy. But, it is still able to give a practical solution as $T_{M C}(q)$ is much smaller than $T_{P}(q)$. In this case, we have $T_{2}=O\left(\sum_{i=1}^{s}\left(T_{P}\left(q_{i}\right)+T_{C R T}\left(q_{1} \cdots q_{i-1}, q_{i}\right)\right.\right.$ $\left.\left.+T_{R}\left(\prod_{j=1}^{i} q_{j}\right)\right)+\sum_{i=1}^{t} T_{M C}\left(q_{i}\right)\right)$, where $\prod_{i=1}^{s} q_{i}=O\left(B_{R}\right)$ and $\prod_{i=1}^{t} q_{i}=O\left(B_{T}\right)$.

Omitting the correctness check in U1, the total efficiency can be much improved. In this case, the result is not proven to be correct, but it will be with a high probability.

We will show certain practicality and theoretical efficiency of the multi-modular strategy, as it can use the both usages of modular check. In Section 4.2, we will give details of algorithms based on multi-modular strategy, and in Section 4.3, based on algorithms given in Section 4.2, we will discuss those efficiency, including estimation on $T_{C}$.

\subsection{Algorithms}

In the case of the computation of the splitting field of the polynomial $f$, the target will be the Gröbner basis $\mathcal{G}$ of the splitting ideal corresponding to the symmetric representation $G$ of the Galois group of $f$. All our first inputs came from the computation of $G_{f}$ by modular algorithm 
(see $[21],[9,10])$ modulo $p_{1}^{k_{1}}$ a power of a prime satisfying property $\mathbb{P}$. From this computation we obtain a finite sequence $\left\{\left(I_{i}, A_{i}\right): i=1, \ldots, t\right\}$ of invariants and their integer evaluation modulo $p_{1}^{k_{1}}$ as in Theorem 3.2. Because, we will use multi-modular strategy $\mathrm{S} 2$ for computing $\mathcal{G}$ we may need to reorder the roots of $f$ modulo $p_{2}^{k_{2}}$, where $p_{2}$ is a prime satisfying $\mathbb{P}$ different from $p_{1}$. To do this we use the sequence $\left\{\left(I_{i}, A_{i}\right)\right\}$ as in Theorem 3.2 , this is described in the following function:

\section{Function: GoodOrdering $\left(\left(p_{2}, k_{2}\right)\right)$}

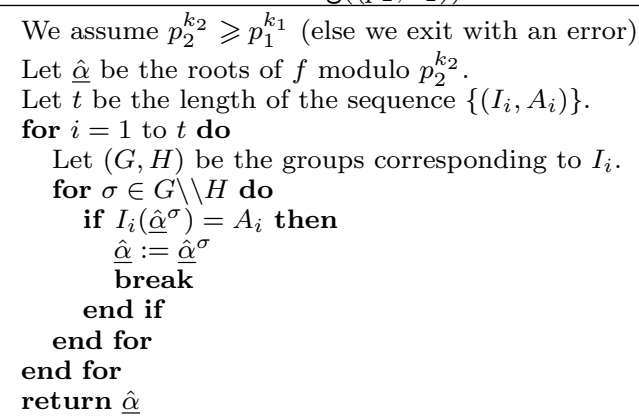

As presented in Section 4.1 we now give an algorithm to compute a candidate $\mathcal{G}_{\text {cand }}=\left\{\tilde{g}_{1}, \ldots, \tilde{g}_{n}\right\}$ for the target $\mathcal{G}$ by using a heuristic bound $B_{T}$. This first step is described by the algorithm Proc_CAND that takes as input an approximation of the roots of $f \bmod p_{1}^{k_{1}}$ as a Gröbner basis $\mathcal{G}^{\left(p_{1}, k_{1}\right)}$ of the splitting ideal of $f \bmod p_{1}^{k_{1}}$, the Galois group $G_{f}$ corresponding to the order of these roots and a set $\mathcal{P}$ of couples $(p, k)$ where $p$ 's are different primes (different from $p_{1}$ ) satisfying property $\mathbb{P}$ and $k$ 's are integers such that $p_{1}^{k_{1}} \prod_{(p, k) \in \mathcal{P}} p^{k} \geq B_{H}$. To the group $G_{f}$ is associated a computation scheme which is represented by the set $\mathcal{I}$ of indexes of the $g_{i}$ to compute in $\mathcal{G}$ and the corresponding $i$-relation $E_{i}$. Before giving this first algorithm, we give a specific Chinese Remaining Theorem procedure which will be used in the sequel:

Procedure: SpecCRT $\left(\left\{g_{i} \bmod M, \quad i \in \mathcal{I}\right\},(p, k)\right)$

Compute and reorder the roots of $f \bmod p^{k}$.

Interpolate the $g_{i}$ 's mod $p^{k}$ corresponding to $E_{i}$ with $i \in \mathcal{I}$. Let $M$ be the modulus $M \times p^{k}$

By CRT compute all the $g_{i} \bmod M(i \in \mathcal{I})$

Now, we can describe the first algorithm corresponding to the first step Proc_CAND:

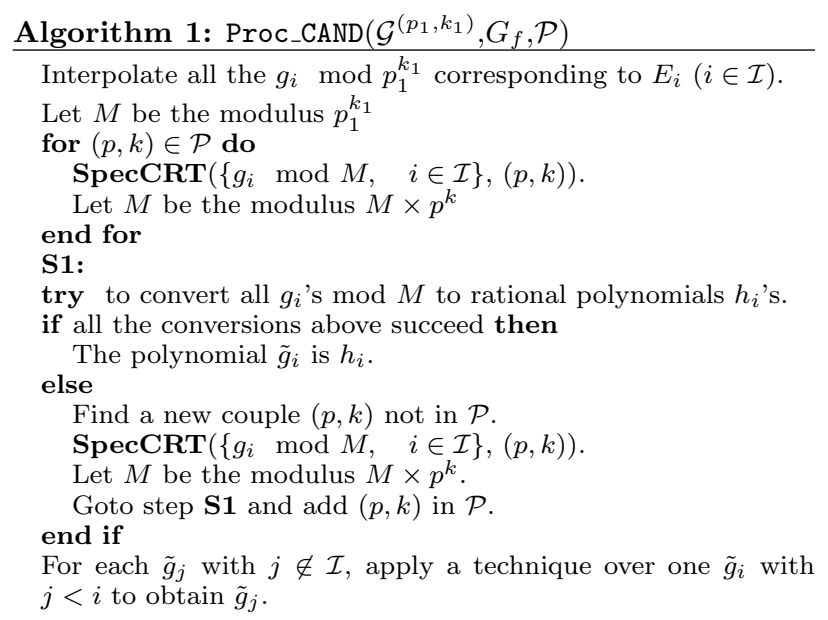

Return $\mathcal{G}_{\text {cand }}, G_{f}, \mathcal{I}, \mathcal{P}$

Since we concentrate here on multi-modular strategy for the computation of $\mathcal{G}$, we give an algorithm corresponding to Proc_CHECK based on the strategy S2. Recall that this algorithm is based on the ideal inclusion test given in [18]:

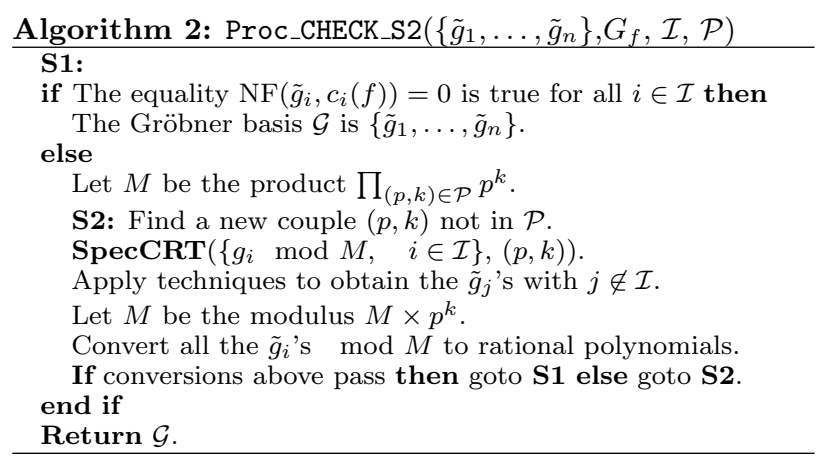

As we said in Section 4.1, we can take advantage of the multi-modular strategy in Proc_CHECK too. Here we give the implementation of Proc_MODCHECK with a generic stopping condition which can be stated in function of the chosen U1 or U2 version.

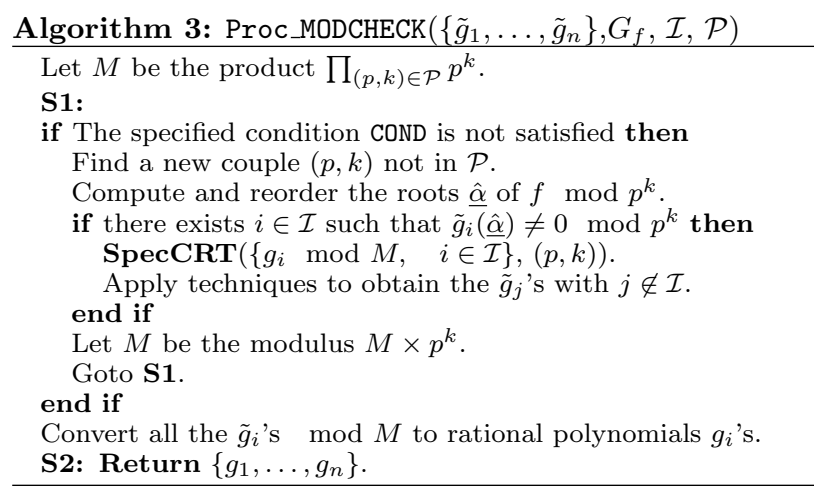

If condition COND is chosen to be the one corresponding to the version U1 then the output of Proc_MODCHECK is not proven but we can use it as an input of Proc_CHECK. Otherwise, the condition COND will correspond to version U2 and in this case the output will be proven.

\subsection{Discussion on Efficiency}

Here we discuss the efficiency of the proposed "multimodular strategy" along by basic estimation on concrete procedures given in the previous subsection. We use the facts given in [18]. By actual experiments on real computers, the authors found that sometimes the correctness check dominated the total efficiency, even though the modulus used there was the same order of the real bound. To resolve this problem, we introduce the usage U2 and show that it can give good estimation on the total efficiency.

To estimate the total efficiency, we have to give concrete representation of the functions $T_{P}, T_{R}, T_{C R T}, T_{H}, T_{M C}$ and $T_{C}$. To distinguish the time for Step 1-1 of Proc_CAND by linear system solving [18] and that by Lagrange interpolation in Section 3.4, we denote by $T_{P L}$ the time by linear system solving and by $T_{P I}$ that by interpolation. Also, we denote by $\mathbb{M}(q)$ the unit cost of integer arithmetics of size $q$. Then, $\mathbb{M}(q)=O\left(q^{2}\right)$ for usual multiplication technique, and $\mathbb{M}(q)=O\left(q^{1+\epsilon}\right)$ for fast multiplication technique. Also by 
[18], we have $T_{C}=O\left(\log (n) c\left(G_{f}\right)^{2} \mathbb{M}\left(\log \left(B^{\prime}\right)\right)\right.$ and $B^{\prime}$ is the largest integer appeared in the normal form computation. (Finding a good estimation on $B^{\prime}$ is a difficult problem.)

For the strategy S2, it had better to use Lagrange interpolation for Proc_CAND and $T_{P I}(q)=O\left(c\left(G_{f}\right)^{2} \mathbb{M}(\log (q))\right)+$ $T_{G O}$, where $T_{G O}$ is the time for GoodOrdering. For the two quantities $T_{C R T}(A, B)$ and $T_{R}$, we can apply fast extended GCD computation technique for Chinese remainder Theorem and rational reconstruction (see [15]), and thus

$T_{C R T}(A, B)=O\left(c\left(G_{f}\right) \mathbb{M}(\log (C)) \log \log (C)\right)$, where $C=$ $\max \{A, B\}$ and $T_{R}(q)=O\left(c\left(G_{f}\right) \mathbb{M}(\log (q)) \log \log (q)\right)$.

As to the modular check of correctness, we divide each candidate $\tilde{g}_{i}$ by $q=p^{k}$ and substitute the p-adic approximation of roots of $f$, and so $T_{M C}(q)=O\left(c\left(G_{f}\right)(\mathbb{M}(\log (q))+\right.$ $\mathbb{M}(\log (D)))$, where $D$ is the maximal absolute value of numerators and denominators of coefficients of $\tilde{g}_{i}$ 's. As $D<$ $B_{R}$, we have $T_{M C}(q)=O\left(c\left(G_{f}\right)\left(\mathbb{M}\left(\log \left(B_{R}\right)\right)\right)\right.$.

For the strategy $\mathrm{S} 3$, by the estimation in [18], we have $T_{P L}(q)=O\left(\left(c\left(G_{f}\right)^{\omega} \mathbb{M}(\log (q))\right)\right.$, and $\sum_{i=2}^{s} T_{H}\left(q_{i}, q_{i-1}\right)=$ $O\left(n^{2} c\left(G_{f}\right)^{2} \mathbb{M}\left(\log \left(q_{s}\right)\right)\right)$ where $\omega$ represents a feasible matrix multiplication exponent and $2 \leq \omega \leq 3$ (see [8]).

Thus, accumulating all representations, the total cost can be estimated in slightly rough but simple form, where we are assuming that $\log \left(B_{T}\right)$ is much larger than $\log \left(B_{R}\right)$ and the size of modulus $q$ used is close to the size of the real bound $B_{R}$, that is, the number of correctness checks in U1 and that of CRT construction in U2 can be bounded (one or very small).

Proposition 4.1. Assume that $\log \left(B_{T}\right)$ is much larger than $\log \left(B_{R}\right)$ and the size of modulus $q$ used and that of $B_{R}$ are the same order.

(1) When we use the modular check U1, we have

$$
\begin{aligned}
& T_{2}=O\left(c\left(G_{f}\right)^{2} \mathbb{M}\left(\log \left(B_{R}\right)\right)\right.+c\left(G_{f}\right) \mathbb{M}\left(\log \left(B_{R}\right)\right) \log \log \left(B_{R}\right) \\
&\left.+T_{G O}+T_{C}\right), \\
& T_{3}=O\left(c\left(G_{f}\right)^{\omega} \mathbb{M}\left(\log \left(q_{1}\right)\right)+n^{2} c\left(G_{f}\right)^{2} \mathbb{M}\left(\log \left(B_{R}\right)\right)+T_{C}\right),
\end{aligned}
$$

where $T_{C}=O\left(\log (n) c\left(G_{f}\right)^{2} \mathbb{M}\left(\log \left(B^{\prime}\right)\right)\right.$ and $B^{\prime}$ is the largest integer appeared in the normal form computation.

(2) When we use the modular check U2, we have

$$
\begin{gathered}
T_{2}=O\left(c\left(G_{f}\right)^{2} \mathbb{M}\left(\log \left(B_{R}\right)\right)+c\left(G_{f}\right) \mathbb{M}\left(\log \left(B_{R}\right)\right) \log \log \left(B_{R}\right)\right. \\
\left.+c\left(G_{f}\right) \mathbb{M}\left(\log \left(B_{T}\right)\right)+T_{G O} \frac{\log \left(B_{T}\right)}{\log \left(B_{R}\right)}\right) .
\end{gathered}
$$

Taking account of the estimation on $B_{T}$ in Section 3.5, when GoodOrdering can be efficiently done like as $T_{G O}$ is $O\left(c\left(G_{f}\right) \mathbb{M}\left(\log \left(B_{R}\right)\right)\right)$, the strategy S2 with the modular check $\mathrm{U} 2$ can give the most efficient computation.

\section{EXPERIMENTS AND REMARKS}

We have implemented the algorithms of Section 4.2 with the computer algebra system MAGMA (version 2.13) in the case of an irreducible monic integral polynomial. This version of MAGMa provides a lot of functions for using the byproduct of the modular computation of the Galois group. All these functions are very efficient and easy to use but we need a little more, thus we have rewritten a large part of our sub-functions in order to be more efficient.

Reordering the roots: In the case of the normalizer of $G_{f}$ in $S_{n}$ is reduced to $G_{f}$, it is sometimes better to recompute the Galois group $G_{2}$ of $f$ using another prime $p_{2}$ and we finally reorder the roots by applying the permutation which conjugates $G_{f}$ and $G_{2}$, as we can use the efficient computation of the Galois group. This is specially effective when the descending process in the subgroups tree encounter two succeeding groups with large index. This method could be generalized by considering the normalizer of $G_{f}$ and will study in a future implementation.

Choice of the primes $p$ : Because of the limit imposed by the Tchebotarev's density theorem it may be hard to find primes which split completely in the stem field defined by the polynomial $f$. On the other hand, the costs of the padic arithmetic increase according to the order of the Galois group of $f$ modulo $p$. Thus, we choose primes satisfying the property $\mathbb{P}$ such that the Galois group of $f$ modulo theses primes has an order of at most 2.

Heuristic bound $B_{H}$ : We choose, as an heuristic, to begin the computation Proc_CAND modulo the fifth power of the product of one, two or three minimal primes satisfying the condition state before.

Comments on the experiments: We try our implementation on several polynomials given by [11] from degree 6 to 9 and some polynomials of greater degree (not more than 13) corresponding to interesting computation schemes. By using this heuristic bound, Proc_CAND already computes in all the cases the final result, thus the remaining computation is the check procedure without any reconstruction. For groups $G$ with small order or $c(G)$ (say under 500) our new implantation will not give a really better efficiency than the one presented in [18] which was already very efficient. The only gain is given by the use of the interpolation in place of the linear system resolution. Moreover, if the group is very small in the symmetric group of same degree, the reordering procedure may be time consuming. Thus, in this case it is preferable to use mono-modular algorithm and check the result by normal forms computations (Proc_CHECK_S2). In the case of groups $G$ with high order and small $c(G)$, our experiments show that by using interpolation, the gain of efficiency of this new implementation is comparable to the gain of theoretical complexity. In these cases, the experimental cost of the reordering of the roots and the reconstruction of the polynomials by Chinese remainder theorem are very small in comparison to the other parts of the computation. Concerning the check procedure, in these cases, the modular strategy with version U2 is always better than the procedure Proc_CHECK_S2. This experiments allow us to think that this new method should be use for the computation of splitting fields with high absolute degree, the remaining bottleneck is the efficient calculation of a computation scheme (we have tabulated them up to degree 10).

Remarks on parallel computations: The computer algebra system MAGMA do not integrate any possibility for parallel computation, thus we have implemented all our algorithms with a sequential style. But, during our experiments we reported that all the parts of the computation that can be shared on different cores have the same time consuming in general. Thus, implementing these algorithms in a parallel system will have a real impact on the efficiency. For example, in the case of Proc_CAND the computations modulo two different primes has almost the same time consuming in general (this is the case when the word sizes of these primes are the same and they have the same decomposition property modulo $f$ ). 
We give here some specific examples and comments about them. We give, for each example, the name of the group $G$ in Butler and McKay's nomenclature, the order of $G$ and the integer $c(G)$ (as the sum of the $i$-relations degrees). The column Primes shows the timings of computing primes with good properties. The column Lin. shows timing Proc_CAND using linear systems solving as in [18], Int. those when we use interpolation, S2 the timings for check procedure Proc_CHECK_S2, U2 the timing of Proc_MODCHECK based on version U2 and Ord. the timing of reordering the roots. The total timings can be obtained by summing the column corresponding to strategies used during the computation. The measurements were made on a personal computer with a $3.0 \mathrm{GHz}$ Intel Xeon 64bits (all the timings are given in seconds).

\begin{tabular}{|c|c|c|c|c|c|c|c|c|}
\hline Group & $|G|$ & $c(G)$ & Primes & Lin. & Int. & S2 & U2 & Ord. \\
\hline \hline $10 T_{39}$ & 3840 & 10 & 2.13 & 0.04 & 0.05 & 0.01 & 0.01 & 1.01 \\
$10 T_{36}$ & 1920 & $960+10$ & 0.81 & 141.2 & 60.5 & 55.4 & 2.1 & 1.3 \\
$9 T_{32}$ & 1512 & $1512+1512$ & 0.57 & 540.8 & $65.1+67.3$ & 412.1 & 4.1 & 0.5 \\
$9 T_{29}$ & 648 & $18+648$ & 0.67 & 40.1 & 9.3 & 1.22 & 1.5 & 1.0 \\
$9 T_{25}$ & 324 & 27 & 0.08 & 6.54 & 7.1 & 0.2 & 0.5 & 2.4 \\
$8 T_{48}$ & 1344 & 336 & 1.25 & 9.89 & 4.23 & 15.34 & 1.1 & 0.8 \\
$7 T_{6}$ & 2520 & 2520 & 1.5 & 785.3 & $76+157.9$ & 8.2 & 2.1 & 2.5 \\
\hline
\end{tabular}

The first line shows a very special case where the total timing is dominated by the research of primes with good property. Theses cases appear when the group is very large in comparison with its size $c(G)$ as here. On the last line, we show the two different timings for computing modulo two different primes. In this case, the first one corresponds to a prime which splits completely the polynomial $f$ in contrary to the second one. This is a general fact, for a fixed word size, using a splitting prime will give better timings (in this case the first one is 10037 the second is 53). The remaining examples show that even the times between the different strategies are comparable, using multi-modular and interpolation approaches are in general better. We did not compare these timings with the ones given in [13] because of the difference of architecture, but if we project them on the same computer our implementation would be more efficient with a factor from 10 to 1000 (this big difference may come from the fact that computation schemes are not used in [13]). Also, we did not integrate in the last table the timings for computing $\mathcal{G}$ in MAGMA (version 2.14) with the new function GaloisSplittingIdeal (with parameter Roots set to false) since we can only obtain two of them by waiting not more than 300 seconds.

\section{CONCLUSION}

We have presented a new method based on a multi-modular strategy for the computation of the splitting field of a polynomial $f$. This new method is a good compromise for the one presented in [18] since it gives better results in the case where the later was inefficient. Also, the experiments show that this multi-modular method is a good candidate for a parallel implementation on a multi-core architecture.

The reordering roots function we give here is a general one. We plan to integrate efficient techniques of Galois groups computation (like the ones presented in [10]) in order to reduce the length of the descending branch in the subgroups tree.

We hope that such a multi-modular method could be generalized to the problem of computing algebraic or integral relations between the roots of a polynomial like in [7].

\section{ACKNOWLEDGMENT}

The authors would like to thank the referees for their valuable remarks.

\section{REFERENCES}

[1] I. Abdeluaouad, S. Orange, G. Renault, and A. VAlibouze. Computation of the decomposition group of a triangular ideal. A AECC Journ., 15(3-4):279-294, 2004.

[2] J.-M. Arnaudiès And A. VAlibouze. Lagrange resolvents. J. Pure Appl. Algebra, 117/118:23-40, 1997. Algorithms for algebra (Eindhoven, 1996).

[3] Anai, H., Noro, M., And Yokoyama, K. Computation of the splitting fields and the Galois groups of polynomials. In Algorithms in algebraic geometry and applications, vol. 143 of Progr. Math. Birkhäuser, Basel, 1996, 29-50.

[4] Becker, T., And Weispfenning, V. Gröbner bases, vol. 141 of Graduate Texts in Mathematics. Springer-Verlag, New York, 1993.

[5] Bosma, W., Cannon, J., and Playoust, C. The Magma algebra system. I. The user language. J. Symbolic Comput. 24, 3-4 (1997), 235-265.

[6] Dahan, X., And Schost, É. Sharp estimates for triangular sets. In ISSAC '04: Proc. of the 2004 International Symposium on Symbolic and Algebraic Computation (New York, 2004), ACM Press, pp. 103-110.

[7] Fieker, C. AND GRAAF, W. Integral linear dependencies of algebraic numbers and algebraic Lie algebras. $L M S J$. Comput. Math., 10 (2007), 271-287

[8] J. VON ZUR Gathen AND J. Gerhard. Modern computer algebra. Cambridge University Press, Cambridge, second edition, 2003

[9] GeIssLeR, K. Berechnung von Galoisgruppen über Zhalund Funktionenkörpern. PhD thesis, Univ. Berlin, 2003.

[10] GeIssler K. And KlÜNers, J. Galois group computation for rational polynomials. J. Symbolic Comput., 30(6):653-674, 2000.

[11] Klüners, J., ANd Malle, G. A database for field extensions of the rationals. LMS J. Comput. Math. 4 (2001), 182-196 (electronic).

[12] LAzARD, D. Solving zero-dimensional algebraic systems. $J$. Symbolic Comput. 13, 2 (1992), 117-131.

[13] Lederer, M., Explicit constructions in splitting fields of polynomials. Riv. Mat. Univ. Parma (7), 3* (2004), 233-244.

[14] MCKay, J., And Stauduhar, R. Finding relations among the roots of an irreducible polynomial. In Proceedings of the 1997 International Symposium on Symbolic and Algebraic Computation (New York, 1997), ACM, pp. 75-77.

[15] PAN, V. AND WANG, X. Acceleration of Euclidean Algorithm and Rational Number Reconstruction. SIAM J. Comput. Vol.32, No.2, pp.548-556, 2003.

[16] M. Pohst and H. Zassenhaus. Algorithmic Algebraic Number Theory. Cambridge Univ. Press, Cambridge, 1989.

[17] Renault, G. Computation of the splitting field of a dihedral polynomial. In Proc. of the 2006 International Symposium on Symbolic and Algebraic Computation (New York, 2006). ACM Press, pp. 290-297.

[18] Renault, G., and Yokoyama, K. A modular method for computing the splitting field of a polynomial. In Proc. of the 7th Algorithmic Number Theory Symposium ANTS-VII, Berlin, Germany, 2006, LNCS 4076, Springer, pp. $124-140$.

[19] N. Rennert and A. Valibouze. Calcul de résolvantes avec les modules de Cauchy. Exp. Math., 8(4):351-366, 1999.

[20] Tchebotarev, N. Gründzüge des Galois'shen Theorie. P. Noordhoff, 1950.

[21] Yokoyama, K. A modular method for computing the Galois groups of polynomials. J. Pure Appl. Algebra $117 / 118$ (1997), 617-636. 